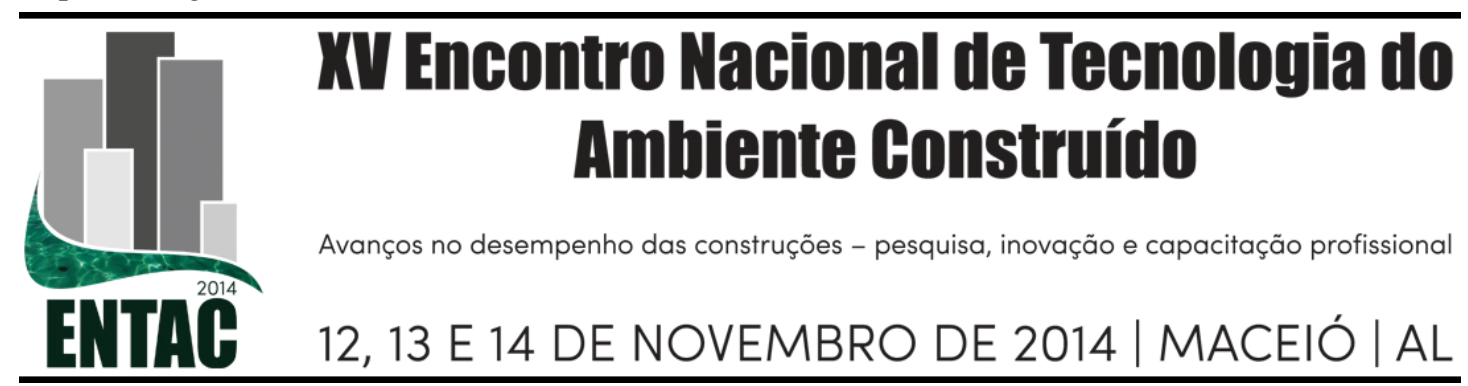

\title{
LEVANTAMENTO DE MANIFESTAÇÕES PATOLÓGICAS EM ESTRUTURAS DE CONCRETO ARMADO NO ESTADO DO CEARÁ
}

\author{
SILVA, Luiza Kilvia da (1); CABRAL, Antonio Eduardo Bezerra (2)
}

(1) Engenheira Civil, GPMATE/UFC (2) Professor Doutor, Grupo de Pesquisa em Materiais de Construção e Estruturas (GPMATE), eduardo.cabral@ufc.br

\begin{abstract}
RESUMO
O Brasil é um país que convive com diversos problemas sociais (desemprego, saúde, educação, habitação, entre outros) e está em fase de desenvolvimento econômico, assim, não pode se dá ao luxo de custear despesas extremamente elevadas em obras de reparo estrutural, as quais, na maioria das vezes, poderiam ser evitadas. É nessa perspectiva que a pesquisa neste trabalho realizada busca levantar dados das principais manifestações patológicas incidentes nas estruturas de concreto armado no Estado do Ceará, utilizando como banco de dados arquivos oriundos da Divisão de Materiais (DIMAT) do Núcleo de Tecnologia Industrial do Ceará (NUTEC) e trabalhos realizados por alunos da Universidade Federal do Ceará (UFC) para a disciplina Patologia e Recuperação de Estruturas de Concreto. Foram analisadas manifestações patológicas em 30 obras, estas distribuídas quanto à forma de uso em: residenciais, comerciais e públicas; e de acordo com a área de entorno a qual está inserida: salina, urbana, industrial ou rural. A partir de tais dados, obteve-se o percentual de ocorrência das principais manifestações patológicas, observando que as estruturas apresentaram degradação principalmente devido à ação da corrosão de armaduras e das fissuras, sendo que tais manifestações estão diretamente associadas ao estabelecimento de procedimentos inadequados nas etapas de projeto e execução do processo construtivo.
\end{abstract}

Palavras-chave: Manifestações patológicas, concreto armado, Ceará.

\begin{abstract}
Brazil is a country that lives with many social problems (unemployment, health, education, housing, among others) and is being economical as well, development can not afford to pay extremely high costs in structural repair works, which, in most cases, can be avoided. It is in this perspective that the research conducted in this paper seeks to collect data of the main pathological manifestations incidents in reinforced concrete structures in the state of Ceará , using as database files originating from the Division of Materials ( DIMAT ) from the Industrial Technology of Ceará (NUTEC) and work conducted by students of the Federal University of Ceará (UFC) for Pathology and Recovery of Concrete Structures discipline. Distributed these pathological manifestations were analyzed in 30 works, how to use: residential, commercial and public; and according to the surrounding area which is inserted: saline, urban, industrial or rural area. From these data, we obtained the percentage of occurrence of the major pathological manifestations, noting that the structures showed degradation mainly due to the action of reinforcement corrosion and cracks, and such events are directly associated with the establishment of improper procedures in steps design and execution of the construction process.
\end{abstract}

Keywords: Pathological manifestation, reinforced concrete, Ceará 


\section{INTRODUÇÃO}

A NBR 6118 (ABNT, 2007) destaca como mecanismos preponderantes de deterioração do concreto a lixiviação por ação de águas puras, carbônicas agressivas ou ácidas, que dissolvem e carreiam os compostos hidratados de pasta de cimento; a expansão por ação de águas e solos que estejam contaminados com sulfatos, dando origem a reações expansivas e deletérias; a expansão por ação das reações entre os álcalis do cimento e certos agregados reativos; e as reações deletérias superficiais de certos agregados, decorrentes de transformações de produtos ferruginosos presentes na sua constituição mineralógica. Quanto aos mecanismos preponderantes de deterioração relativos à armadura, destacam-se a despassivação por carbonatação e a despassivação por elevado teor de cloreto.

Vários estudos já foram realizados (DAL MOLIN, 1988; SILVA, TRISTÃO e MACHADO apud ARANHA, 1994; SANTAVA apud ARANHA, 1994; ANDRADE, 1997; CARMONA e MEREGA, 1988; NINCE, 1996; ARANHA, 1994) diagnosticando as principais manifestações patológicas apresentadas em vários lugares do Brasil, contudo nunca nenhum levantamento semelhante foi realizado no estado do Ceará.

Assim, buscou-se um levantamento das manifestações patológicas no Estado do Ceará e suas origens para se verificar as causas mais incidentes de deterioração e, assim, alertar o meio técnico para que se tomem medidas preventivas, objetivando ter obras duráveis. Para tanto, foram compilados vários conceitos relacionados ao tema estudado. Ressaltase que este trabalho não tem a ambição de englobar todos os aspectos que têm influência significativa na durabilidade das estruturas, mas sim apresentar alguns pontos relevantes sobre cada um deles, sempre buscando relacionar ao máximo as considerações de caráter tecnológico com as da prática executiva, objetivando apresentar conceitos básicos para a obtenção de obras duráveis.

Portanto, este trabalho tem como objetivo a realização de um levantamento das principais manifestações patológicas que ocorrem nas estruturas de concreto armado no Estado do Ceará.

\section{METODOLOGIA}

A metodologia adotada para o cumprimento da pesquisa foi dividida em algumas atividades interdependentes, sendo estas: levantamento bibliográfico, montagem do banco de dados, eleição das principais manifestações patológicas e análise dos resultados.

O levantamento bibliográfico consistiu no estudo de bases teóricas para facilitar o entendimento sobre o tema estudado como teses, dissertações, monografias, artigos, livros, manuais, revistas, meios eletrônicos, entre outras fontes que forneceram embasamento para o trabalho. Coletado esse material, foi realizada a leitura das bibliografias obtidas, objetivando captar definições e terminologias necessárias para a elaboração deste trabalho.

Foi montado um banco de dados a partir de consultas aos arquivos oriundos da Divisão de Materiais (DIMAT) do Núcleo de Tecnologia Industrial do Ceará (NUTEC) e, também, através de trabalhos realizados por alunos da Universidade Federal do Ceará (UFC) para a disciplina Patologia e Recuperação de Estruturas de Concreto. Esses arquivos foram analisados e, retiraram-se, assim, informações de laudos técnicos de vistorias, projetos de reparo e/ou reforço estrutural. 
Foram analisadas ao todo 30 obras executadas em concreto armado no Estado do Ceará que apresentaram algum tipo de manifestação patológica em sua estrutura entre o período de 2000 a 2011. Ressalta-se que não foi possível catalogar um número maior de obras devido à dificuldade de se ter acesso a laudos e também pelo fato de que muitas estruturas, que apresentam algum tipo de manifestação patológica, são reparadas pelos usuários sem que sejam consultados técnicos especializados, não se tendo, assim, o correto acompanhamento da recuperação e consequentemente a elaboração de laudos.

As obras foram catalogadas segundo as seguintes informações: Forma de Uso (residencial, comercial, industrial ou institucional, pública); Entorno (área salina, área urbana, periferia urbana, área rural ou área industrial); ressaltando-se que não foi verificado o efeito da superposição de diferentes tipos de meio ambiente, devido à dificuldade de se realizar essa determinação.

A classificação do entorno adotada para o Estado do Ceará foi a apresentada por Andrade (1997) em seu trabalho de levantamento de manifestações patológicas nas estruturas no Estado de Pernambuco, uma vez que a maioria das capitais dos estados que compõem a Região Nordeste do Brasil estão localizadas na orla marítima, onde a ação de substâncias agressivas presentes na atmosfera, aliada à ocorrência de altas temperaturas médias juntamente com o teor de umidade elevado, torna o ambiente propício para o desenvolvimento de uma grande variedade de processos de degradação nas estruturas.

Vale destacar que, ao se tomar como referência a cidade de Fortaleza e classificando-a com relação ao Brasil, chega-se à conclusão que todas as obras existentes em tal capital estão inseridas na área salina, em função da própria localização da mesma. Porém, objetivando-se verificar a influência do entorno na distribuição das diferentes formas de degradação nas obras dentro do Estado do Ceará, classificou-se o mesmo em regiões a partir da orla marítima, conforme mostrado no Quadro 1.

Quadro 1 - Classificação do entorno do Estado do Ceará.

\begin{tabular}{|c|c|}
\hline ENTORNO & LOCALIZAÇÃO \\
\hline ÁREA SALINA & $0-1 \mathrm{~km}$ do litoral \\
\hline ÁREA URBANA & $1 \mathrm{~km}-6 \mathrm{~km}$ do litoral \\
\hline ÁREA RURAL & $>15 \mathrm{~km}$ do litoral \\
\hline ÁREA INDUSTRIAL & Obras localizadas na atmosfera industrial \\
\hline
\end{tabular}

Para a definição das manifestações patológicas mais incidentes nas edificações, analisaram-se os resultados dos trabalhos de levantamento das manifestações patológicas nas estruturas de concreto armado feitos no Rio Grande do Sul (DAL MOLIN, 1988), no Espírito Santo (SILVA, TRISTÃO e MACHADO apud ARANHA, 1994), em Santa Catarina (SANTAVA apud ARANHA, 1994), em Pernambuco (ANDRADE, 1997), na Região Sudeste (CARMONA \& MEREGA, 1988), na Região Centro-Oeste (NINCE, 1996) e na Região Norte (ARANHA, 1994). As principais manifestações patológicas encontradas nestes trabalhos foram: fissuras, infiltrações, corrosão de armaduras, desagregação, segregação, manchamento superficial, deformações excessivas, eflorescências e presença de fungos.

As manifestações patológicas do banco de dados foram catalogadas para cada uma das 30 obras em análise, verificando a presença ou não de cada uma das manifestações patológicas a anteriormente citadas. Ao final calculou-se a porcentagem de incidência de cada manifestação em relação ao grupo amostral. 


\section{RESULTADOS}

\subsection{Caracterização do banco de dados}

A distribuição da forma de uso de cada obra classificou-se em pública (21 correspondente a $70 \%$ do total), residencial ( $7-23,3 \%$ do total) e comercial $(2-6,7 \%$ do total), conforme apresentado na Figura 1-a. Nenhuma das obras que compuseram a amostra classificou-se como industrial.

Quanto à área de entorno das obras catalogadas, sobressaíram-se as áreas salinas e urbanas, correspondentes respectivamente a 43,3\% (13 obras) e 33,3\% (10 obras) do total, e, por conseguinte, a área rural (06 obras - 20\%) e industrial (01 - 3,3\%). A síntese da área do entorno está apresentada na Figura 1-b.

\section{Figura 1 - a) Distribuição da forma do uso das obras catalogadas;}

b) Distribuição da área do entrono das obras catalogadas.
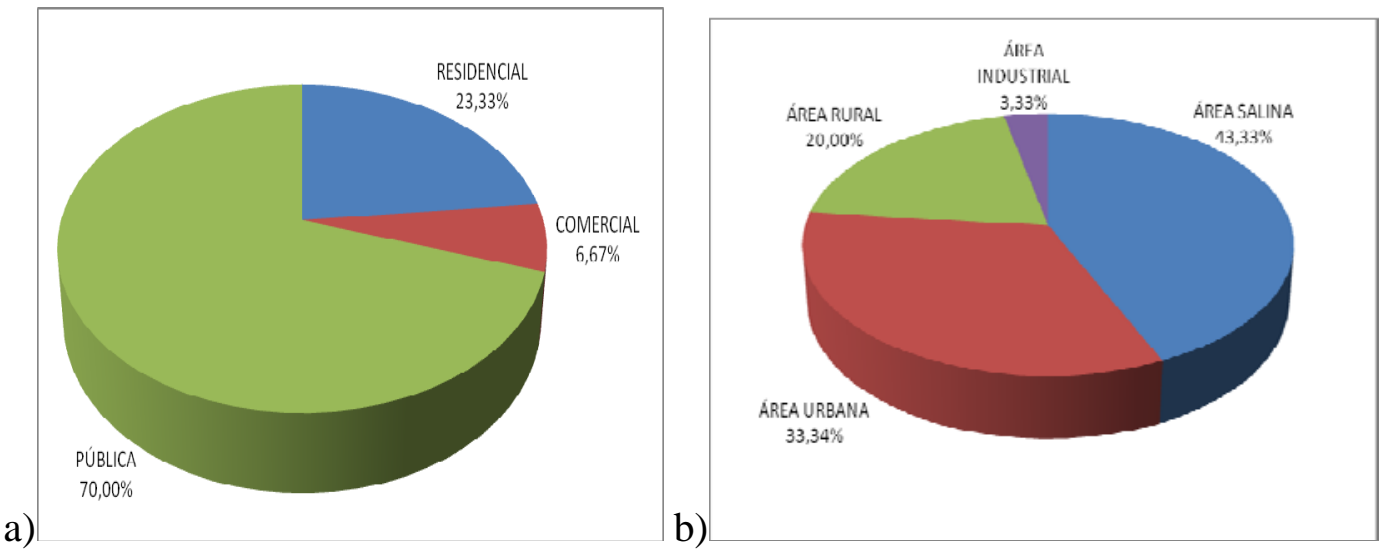

Observa-se que a maior quantidade de obras coletadas está localizada na área salina, com um índice de 43,3\%, seguido da área urbana, com 33,3\%. Assim, dentro da região delimitada, tem-se que a maioria das estruturas localiza-se onde a ação da névoa salina atinge o mais alto grau de agressividade. Tais índices somados chegam ao valor de 76,7\%, mostrando que, além da condição de exposição extremamente desfavorável, a grande maioria das obras atacadas por algum processo de deterioração está localizada na capital, onde há uma maior concentração das mesmas.

Esse valor é condizente com o encontrado por Andrade (1997) em sua análise dos elementos estruturais mais degradados no Estado de Pernambuco, em sua amostra de 189 obras 83,3\% delas encontrava-se inserida em um ambiente salino ou urbano.

\subsection{Manifestações patológicas encontradas nas edificações avaliadas}

O Quadro 2 apresenta a freqüência das manifestações patológicas encontradas em todas as 30 obras em estudo, com o cálculo no final da porcentagem de incidência de cada manifestação em relação ao grupo amostral.

É importante observar no levantamento dos dados que ocorre, em várias situações, uma superposição de manifestações e causas, resultando em um percentual total acumulativo maior que 100\%. A Figura 2 apresenta os índices referentes às manifestações patológicas relatadas, em um universo de 30 obras. 


\section{Quadro 2 - Manifestações patológicas encontradas nas edificações avaliadas}

\begin{tabular}{|c|c|c|c|c|c|c|c|c|c|}
\hline $\begin{array}{l}\text { MANIFESTAÇÃO/ } \\
\text { LOCAL }\end{array}$ & 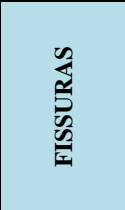 & 聓 & 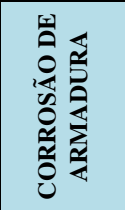 & 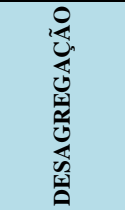 & 选 & 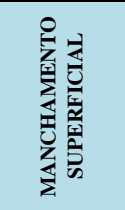 & 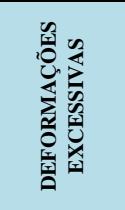 & 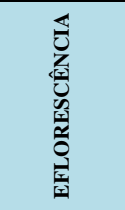 & 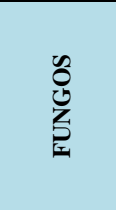 \\
\hline OBRA 1 & $\mathrm{X}$ & - & $\mathrm{X}$ & - & - & $\mathrm{X}$ & - & - & - \\
\hline OBRA 2 & $\mathrm{X}$ & - & $\mathrm{X}$ & - & - & - & - & - & - \\
\hline OBRA 3 & $\mathrm{X}$ & - & $\mathrm{X}$ & $\mathrm{X}$ & - & - & - & $\mathrm{X}$ & - \\
\hline OBRA 4 & $\mathrm{X}$ & $\mathrm{X}$ & $\mathrm{X}$ & - & - & $\mathrm{X}$ & - & - & $\mathrm{X}$ \\
\hline OBRA 5 & $\mathrm{X}$ & $\mathrm{X}$ & $\mathrm{X}$ & $\mathrm{X}$ & - & - & - & $\mathrm{X}$ & - \\
\hline OBRA 6 & $\mathrm{X}$ & $\mathrm{X}$ & $\mathrm{X}$ & $\mathrm{X}$ & - & - & - & - & - \\
\hline OBRA 7 & $\mathrm{X}$ & $X$ & $X$ & - & - & - & - & - & - \\
\hline OBRA 8 & $\mathrm{X}$ & $\mathrm{X}$ & $\mathrm{X}$ & $\mathrm{X}$ & - & $\mathrm{X}$ & $\mathrm{X}$ & - & $\mathrm{X}$ \\
\hline OBRA 9 & $\mathrm{X}$ & - & $\mathrm{X}$ & $\mathrm{X}$ & - & $\mathrm{X}$ & - & $\mathrm{X}$ & $\mathrm{X}$ \\
\hline OBRA 10 & - & - & $X$ & - & - & - & - & - & - \\
\hline OBRA 11 & - & - & $\mathrm{X}$ & - & $\mathrm{X}$ & - & - & - & - \\
\hline OBRA 12 & $\mathrm{X}$ & $\mathrm{X}$ & $X$ & $\mathrm{X}$ & - & - & - & $\mathrm{X}$ & - \\
\hline OBRA 13 & $\mathrm{X}$ & - & $\mathrm{X}$ & - & - & $X$ & - & - & - \\
\hline OBRA 14 & - & - & $\mathrm{X}$ & - & $\mathrm{X}$ & - & - & - & - \\
\hline OBRA 15 & $\mathrm{X}$ & $X$ & $X$ & - & - & $\mathrm{X}$ & - & - & - \\
\hline OBRA 16 & - & $\mathrm{X}$ & $\mathrm{X}$ & $\mathrm{X}$ & - & $\mathrm{X}$ & - & $\mathrm{X}$ & - \\
\hline OBRA 17 & $\bar{X}$ & - & $\mathrm{X}$ & $\mathrm{X}$ & $\mathrm{X}$ & - & - & $\mathrm{X}$ & $\mathrm{X}$ \\
\hline OBRA 18 & - & - & $\mathrm{X}$ & $\mathrm{X}$ & - & - & - & - & - \\
\hline OBRA 19 & $\mathrm{X}$ & - & $\mathrm{X}$ & - & - & - & - & - & - \\
\hline OBRA 20 & $\mathrm{X}$ & - & $X$ & $\mathrm{X}$ & - & - & - & - & - \\
\hline OBRA 21 & $\mathrm{X}$ & $\mathrm{X}$ & $\mathrm{X}$ & $\mathrm{X}$ & $\mathrm{X}$ & $\mathrm{X}$ & $\mathrm{X}$ & $\mathrm{X}$ & $\mathrm{X}$ \\
\hline OBRA 22 & - & - & $X$ & - & $\mathrm{X}$ & - & - & - & - \\
\hline OBRA 23 & $\mathrm{X}$ & - & - & - & - & - & $\mathrm{X}$ & - & - \\
\hline OBRA 24 & $\mathrm{X}$ & - & $X$ & - & - & - & - & - & - \\
\hline OBRA 25 & - & - & $\mathrm{X}$ & $\mathrm{X}$ & - & - & - & - & - \\
\hline OBRA 26 & - & - & $\mathrm{X}$ & $\mathrm{X}$ & - & - & - & - & - \\
\hline OBRA 27 & - & - & $\mathrm{X}$ & $\mathrm{X}$ & - & - & - & - & - \\
\hline OBRA 28 & $\mathrm{X}$ & - & $\mathrm{X}$ & - & $\mathrm{X}$ & - & $\mathrm{X}$ & - & - \\
\hline OBRA 29 & - & $\mathrm{X}$ & $\mathrm{X}$ & - & $\mathrm{X}$ & - & - & $\mathrm{X}$ & - \\
\hline OBRA 30 & $\mathrm{X}$ & - & $\mathrm{X}$ & $\mathrm{X}$ & $\mathrm{X}$ & - & $\mathrm{X}$ & - & - \\
\hline TOTAL & 20 & 10 & 29 & 15 & 8 & 8 & 5 & 8 & 5 \\
\hline$\%$ & $66,67 \%$ & $33,33 \%$ & $96,67 \%$ & $50,00 \%$ & $26,67 \%$ & $26,67 \%$ & $16,67 \%$ & $26,67 \%$ & $16,67 \%$ \\
\hline
\end{tabular}

No levantamento realizado, a manifestação de dano predominante foi a corrosão de armaduras (96,7\% dos casos), associada a um ou mais dos eventos, tais como cobrimento deficiente, fissuras, infiltrações, presença continua de umidade, meio ambiente agressivo e falta ou deficiência de manutenção.

A predominância dessa manifestação já era esperada, pois no Brasil, alguns estudos caracterizaram bem a degradação de estruturas em concreto armado, destacando-se trabalhos como Dal Molin (1988) no Rio Grande do Sul, Aranha (1994) na região Norte, Nince (1996) na região Centro-Oeste e Andrade (1997) em Pernambuco. Nesses trabalhos, a corrosão de armaduras ocupou posição de destaque com valores respectivamente de $12 \%$, 43\%, 30,1\% e 64\% das manifestações encontradas, destaque especial nas regiões de costa, onde a ação dos cloretos como agente de degradação de sobressai.

Silva (2011) em um levantamento da deposição de íons cloretos na cidade de Fortaleza, pelo método da vela úmida, concluiu que esta mantém concentração de cloretos superior a todas as outras capitais da Região Nordeste. A Figura 3 mostra que, enquanto as outras capitais apresentam concentração de cloretos próxima de zero a distâncias de 1000 metros do mar, Fortaleza ainda apresenta uma concentração em torno de $100 \mathrm{mg} / \mathrm{m}^{2}$.dia, destacando, assim, o quão é mais agressiva quando comparada as outras. 
Figura 2 - Incidência das manifestações patológicas nas estruturas de concreto para o Estado do Ceará.

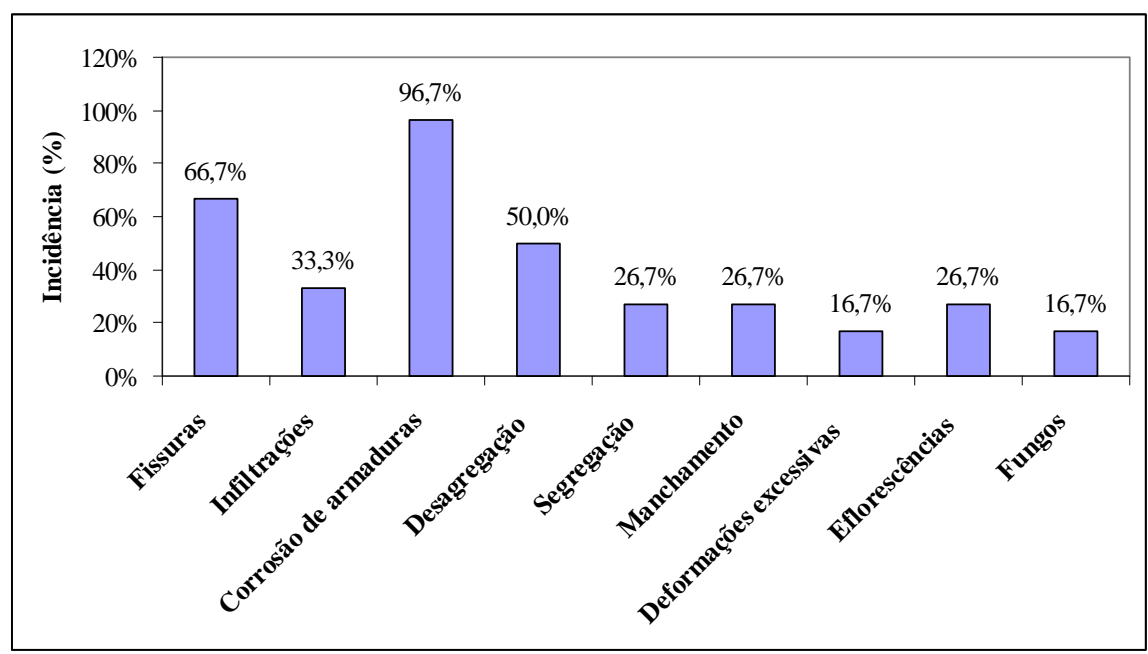

Figura 3 - Teor de cloretos no ar atmosférico de algumas cidades do Brasil

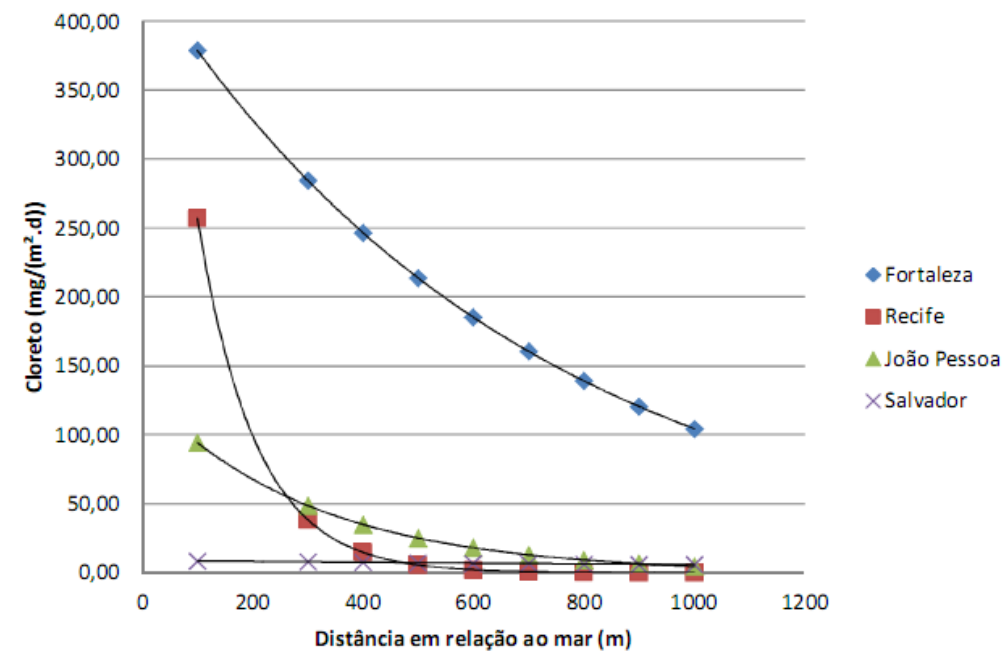

Pamplona e Leal (1982) já alertavam para a agressividade ambiental da cidade de Fortaleza devido à salinidade do mar do Ceará apresentar valores acima da média, aliada aos ventos alísios normais à costa que levam as gotículas de água salgada a vários quilômetros do litoral.

Das obras analisadas, 76,7\% estão localizadas em Fortaleza. Sabendo que a umidade relativa média da cidade normalmente é acima de 70\%, segundo a Fundação Cearense de Metereologia (FUNCEME), tornando o ambiente mais propício para a carbonatação do concreto e facilitando a corrosão eletroquímica nas armaduras, aliado ainda a um baixo índice pluviométrico na cidade, o que aumenta o tempo de permanência do filme de eletrólito nas estruturas (ALBUQUERQUE e OTOCH, 2005), torna-se coerente que a corrosão de armaduras fosse a manifestação patológica mais incidente nas edificações analisadas.

Em seguida, dentre as manifestações patológicas encontradas, problemas com fissuras correspondem a 66,7\% dos casos. Para Souza e Ripper (1998), a fissura pode ser considerada como a manifestação patológica característica das estruturas de concreto, 
sendo mesmo o dano de ocorrência mais comum e aquele que, a par das deformações muito acentuadas, mais chama a atenção dos leigos, proprietários e usuários aí incluídos, para o fato de que algo de anormal está a acontecer.

Pela natureza das estruturas de armado, o aparecimento de fissuras é sinal de que foi excedida a resistência à tração do material e permite investigar, em função de sua tipologia, a origem dos problemas que afetam a estrutura. Por exemplo, nos pilares aparecem fissuras verticais ou ligeiramente inclinadas, se durante a execução ocorreu má colocação, insuficiência e deslocamento dos estribos. Estas fissuras são, neste caso, um sintoma bastante perigoso.

A desagregação do material, que aparece em 50\% dos casos, é um fenômeno que frequentemente pode ser observado nas estruturas de concreto, sendo causada pelos mais diversos fatores, ocorrendo, na maioria dos casos, em conjunto com a fissuração. Esta consiste na perda de massa de concreto devido a um ataque químico expansivo de produtos inerentes ao concreto e/ou devido à baixa resistência do mesmo, caracterizando-se por agregados soltos ou de fácil remoção.

As infiltrações aparecem em 33,3\% dos casos que, segundo Nince (1996), está associado, principalmente, a problemas de projeto (concepção arquitetônica e instalações) e a falta ou deficiência de manutenção.

Os ninhos de concretagem ou segregações aparecem em 26,7\% dos casos avaliados. Estes têm como aspectos gerais vazios na massa de concreto; agregados sem 0 envolvimento da argamassa e concreto sem homogeneidade dos componentes, sendo as suas prováveis causas a baixa trabalhabilidade do concreto, a deficiência no transporte, lançamento e adensamento do concreto e a alta densidade de armaduras. Ou seja, é uma manifestação que está intimamente ligada à execução e supervisão inadequada da concretagem.

As manchas na superfície do concreto apareceram em $16,7 \%$ dos casos. Esta manifestação patológica altera a textura e uniformidade de coloração do concreto causando prejuízos estéticos, podendo causar a instalação de problemas patológicos mais sérios. Os principais fatores que provocam as manchas superficiais são: deficiência dos dispositivos de drenagem; deficiência da vedação nas juntas e degradação química do concreto.

As eflorescências são tipos específicos de manchas de coloração normalmente brancas que surgem frequentemente no concreto e ocorrem devido ao acúmulo de solução saturada de hidróxido de cálcio na superfície do concreto, segundo Helene (2003), podendo ainda formar estalactites nas zonas de maior porosidade do concreto. Para o estudo em questão, as eflorescências apareceram em 16,7\% dos casos avaliados.

A situação de infiltração e umidade na estrutura é um forte estimulador para que microorganismos se proliferem na mesma, implicando no surgimento de manchas esverdeadas denominadas bolor (fungos) que, na pesquisa em especifico, apareceram em $16,67 \%$ das obras avaliadas. Esse problema é agravado principalmente pela falta de manutenção nas estruturas.

As deformações excessivas apareceram em 16,7\% dos casos, sendo estas resultadas de um ou mais dos fatores, tais como problemas de projeto (concepção e/ou detalhamento), mau posicionamento das armaduras na execução e má utilização da estrutura, no que se refere à ação de sobrecarga superior ou incompatível com aquela prevista em projeto. 


\section{CONCLUSÕES}

Em função dos resultados apresentados, a manifestação patológica mais ocorrente foi a corrosão de armaduras, aparecendo em 96,7\% dos casos avaliados. Em função do elevado grau de agressividade ambiental, a corrosão de armaduras encontrou um ambiente totalmente propício para a sua incidência. A origem desta está ligada principalmente a procedimentos inadequados estabelecidos tanto na etapa de projeto, como por meio da falta de especificação de um cobrimento adequado das armaduras e da dosagem do concreto deficiente, quanto na etapa de execução, por falta de cuidado durante a produção da estrutura de concreto.

As fissuras também foram uma manifestação patológica bastante incidente (correspondeu a 66,7\% dos casos). Essas também têm consequências graves na durabilidade das estruturas, pois são veículos de entrada de água e agentes agressivos para o interior da massa de concreto, fazendo com que os fenômenos de degradação, como, por exemplo, a corrosão de armaduras aumente significativamente.

Por meio das análises realizadas pode-se afirmar que a maioria dos danos que ocorreram nas estruturas poderia ser minimizada caso houvesse um efetivo controle da qualidade durante o processo construtivo, aliado a um programa de manutenção preventiva das estruturas de concreto armado.

Ressalta-se que o resultado do levantamento não pode ser tido como absoluto, em razão do caráter, às vezes subjetivo, que envolve a interpretação dos dados, bem como devido ao reduzido número de casos. Porém, informações valiosas foram obtidas e seria bastante oportuno que houvesse um desenvolvimento formal do setor da construção civil no sentido de se patrocinar e padronizar trabalhos desta natureza, objetivando melhorar os níveis atuais de durabilidade e vida útil das estruturas.

\section{REFERÊNCIAS}

Associação Brasileira de Normas Técnicas (ABNT). NBR 6118:2007: Projeto de Estruturas de Concreto - Procedimento. Rio de Janeiro, 2007.

ANDRADE, J.J.O. Durabilidade das estruturas de concreto armado: análise das manifestações patológicas nas estruturas no estado de Pernambuco. Dissertação (Mestrado), Universidade Federal do Rio Grande do Sul, Porto Alegre, 1997.

ANDRADE, T; SILVA,.A.J.C. Patologia das Estruturas. In: ISAIA, Geraldo Cechella. (Ed) Concreto: In.: Concreto: Ensino, Pesquisa e Realizações. Editor: Geraldo Cechella Isaia. São Paulo: IBRACON, 2005, V.1, Cap. 32.

ARANHA, P.M.S. Contribuição ao estudo das manifestações patológicas em estruturas de concreto armado na região amazônica. Dissertação (Mestrado), Universidade Federal do Rio Grande do Sul, Porto Alegre, 1994.

DAL MOLIN, D.C.C. Fissuras em estruturas de concreto armado: análise das manifestações típicas e levantamento de casos ocorridos no estado do Rio Grande do Sul. Dissertação Mestrado em Engenharia - Curso de Pós Graduação em Engenharia Civil, Universidade Federal do Rio Grande do Sul, Porto Alegre,1988.

HELENE, P. Manual de Reparo, Proteção e Reforço de Estruturas de Concreto. Editora Rehabilitar, , São Paulo, 2003.

NINCE, A.A. Levantamento de dados sobre causas de deterioração de estruturas na região Centro-Oeste. Dissertação (Mestrado), Universidade de Brasília, Brasília, 1996 
PAMPLONA, A.D.G.; LEAL, C.R.L.V. Durabilidade do concreto no litoral de Fortaleza. Estudo UFC \& RFFSA, 1982.

SILVA, F. A. S. Avaliação do teor de íons cloreto no ar atmosférico da praia de futuro em Fortaleza/CE. Monografia. Universidade Federal do Ceará, 2011.

SOUZA, V. C.; RIPPER, T. Patologia, recuperação e reforço de estruturas de concreto. São Paulo: Pini, 1998. 